\title{
Book Review \\ "Habiter la ville: évolution démographique et attractivité résidentielle d'une ville-centre"s"
}

\author{
T. Theurillat \\ Université de Neuchâtel, Neuchâtel, Switzerland \\ Correspondence to: T. Theurillat (thierry.theurillat@unine.ch)
}

Rérat, P.: Habiter la ville: évolution démographique et attractivité résidentielle d'une ville-centre, Editions Alphil - Presses universitaires suisses, 566 pp., ISBN-13: 978-2940235-66-7, €42.00, 2010.

Il est courant d'associer l'attractivité renouvelée des villes et les opérations de revitalisation urbaine au phénomène de la "gentrification", qui désigne l'occupation des nouveaux quartiers urbains par les classes aisées de la population. C'est du moins l'un des grands débats qui a marqué la géographie urbaine, en particulier anglo-saxonne, ces 15-20 dernières années.

Patrick Rérat questionne l'attractivité résidentielle des centres villes en Suisse, et plus spécifiquement à Neuchâtel à travers les mutations démographiques et migratoires. Dans ses recherches, l'auteur fait preuve d'originalité à plusieurs égards. Tout d'abord, son approche pluraliste lui a permis de réunir les théories de développement urbain et de la démographie urbaine autour des concepts de mobilité résidentielle, d'étalement urbain et de ré-urbanisation. Le "retour en ville" est ainsi perçu à la fois comme la conséquence de motivations personnelles et collectives des habitants mais aussi comme le fruit de nouveaux marchés immobiliers (la production et l'offre de logements) et d'actions publiques spécifiques. Par ailleurs, ce pluralisme théorique s'enracine empiriquement dans une triangulation méthodologique ambitieuse. L'utilisation de statistiques officielles (population, activités économiques), de questionnaires auprès des habitants et d'entretiens semi-directifs avec les acteurs du marché de la construction et de l'immobilier permettent à Patrick Rérat de décrire de manière approfondie l'évolution démographique et migratoire de la région neuchâteloise entre les années 1970 et 2000. Il met ainsi en évidence le passage d'un régime urbain industriel à un régime urbain métropolitain. Ce dernier est marqué par un regain de l'attractivité des centres villes suisses tel que Neuchâtel au tournant du nouveau siècle. Enfin, l'étude de cas menée à Neuchâtel permet de transposer et de questionner la problématique de la ré-urbanisation et plus précisément de la "gentrification" dans le contexte des villes moyennes, les principales études réalisées à ce jour traitant le plus souvent des grandes métropoles.

Sans tomber dans l'écueil, l'ouvrage propose un débat autour du concept de "gentrification" et autour de son évolution par rapport aux réalités urbaines d'hier et d'aujourd'hui, d'ici et d'ailleurs dans des approches théoriques et méthodologiques multiples. Qui sont alors les "gentrifieurs" neuchâtelois et plus généralement des villes moyennes en $\mathrm{Su}$ isse? Il s'agit principalement, de couples ou de familles avec enfant(s) (pour un tiers) appartement aux classes moyennes et supérieures (CSP+: de formation universitaire) et, à côté des migrants qualifiés (principalement européens), de jeunes adultes. Ces habitants, déjà en ville pour une bonne partie, s'installent au centre-ville pour des raisons pratiques liées à la proximité aux aménités urbaines et aux infrastructures de transport. C'est d'ailleurs à ces catégories que les nouveaux quartiers, de standing assez élevés, construits de 2000 à 2007 à Neuchâtel ont été destinés.

Pour Patrick Rérat, la "new build gentrification" ne marque pas un frein au processus d'étalement à l'échelle régionale, puisque la perspective d'habiter à la périphérie des villes moyennes demeure attractive pour une partie des "gentrifieurs". Outre le fait de documenter les logiques urbaines contemporaines en Suisse, avec l'éclatement des 
espaces de vie (travail, loisirs, habitat) et le rôle de la mobilité/connectivité, cette thèse permet de mieux comprendre les enjeux abordés récemment par l'économie résidentielle (soit de l'attraction d'habitants ou de touristes sur un territoire qui s'ajoute alors à la concurrence territoriale pour attirer des activités économiques) ou encore du renouveau des grands projets urbains en Suisse et de la dynamique des marchés immobiliers. 